\title{
Quality of Life and Work Productivity Improvements with Upadacitinib: Phase 2b Evidence from Patients with Moderate to Severe Crohn's Disease
}

\author{
Laurent Peyrin-Biroulet • Edouard Louis · Edward V. Loftus Jr. • \\ Ana Lacerda · Qian Zhou · Yuri Sanchez Gonzalez · Subrata Ghosh
}

Received: December 21, 2020 / Accepted: February 8, 2021 / Published online: March 23, 2021

(C) The Author(s) 2021

\begin{abstract}
Introduction: In the phase 2 CELEST study, positive efficacy results were obtained with the Janus kinase 1 inhibitor upadacitinib for adult patients with moderate to severe Crohn's disease. We present the health-related quality of life and work productivity improvement results with upadacitinib from CELEST.
\end{abstract}

Methods: CELEST (NCT02365649) was a double-blind study where patients were randomized 1:1:1:1:1:1 in the 16-week induction period to placebo or upadacitinib $3 \mathrm{mg}$ twice

Supplementary Information The online version contains supplementary material available at https:// doi.org/10.1007/s12325-021-01660-7.

L. Peyrin-Biroulet

Department of Gastroenterology, Nancy University

Hospital, Nancy, France

L. Peyrin-Biroulet $(\square)$

Université de Lorraine, Nancy, France

e-mail: peyrinbiroulet@gmail.com

E. Louis

University Hospital CHU of Liège, Liège, Belgium

E. V. Loftus Jr.

Mayo Clinic College of Medicine, Rochester, MN, USA

A. Lacerda · Q. Zhou · Y. Sanchez Gonzalez

AbbVie Inc., North Chicago, IL, USA

S. Ghosh

University of Birmingham, Birmingham, UK daily (BID), $6 \mathrm{mg}$ BID, $12 \mathrm{mg}$ BID, $24 \mathrm{mg}$ BID, or $24 \mathrm{mg}$ once daily (QD). Patients completing the induction period were re-randomized $1: 1: 1$ to receive upadacitinib $3 \mathrm{mg} \mathrm{BID}, 12 \mathrm{mg} \mathrm{BID}$, or $24 \mathrm{mg}$ QD for 36 weeks or $3 \mathrm{mg}$ BID, $6 \mathrm{mg}$ BID, or $12 \mathrm{mg}$ BID (after amendment). Inflammatory Bowel Disease Questionnaire (IBDQ), European Quality of Life-5 Dimensions visual analog scale (EQ-5D VAS), and Work Productivity and Activity Impairment (WPAI) questionnaire outcomes were assessed at baseline and Weeks 8,16 , and 52.

Results: At Week 16, a significant percentage $(P \leq 0.05)$ of patients receiving upadacitinib 6-mg BID dose or higher achieved IBDQ response (IBDQ score change $\geq 16$ points; $49 \%-57 \%$ for upadacitinib vs. $24 \%$ for placebo) and IBDQ remission, except $24 \mathrm{mg}$ QD (IBDQ score $\geq 170 ; 26 \%-39 \%$ for upadacitinib vs. $11 \%$ for placebo). Greater improvements in IBDQ total score, EQ-5D VAS, and activity impairment from baseline $(P \leq 0.1)$ versus placebo were also observed. Larger improvements $(P \leq 0.1)$ in IBDQ response and total score and EQ-5D VAS were observed at Week 8 with 6 and $24 \mathrm{mg}$ BID versus placebo, with improvements for all dosages maintained or greater at Week 52 for IBDQ, EQ-5D VAS, and WPAI endpoints, in particular for the 12-mg BID group.

Conclusion: Improvements in health-related quality of life and work productivity were achieved and sustained with upadacitinib in 
patients with moderate to severe Crohn's disease.

Trial Registration: ClinicalTrials.gov identifier, NCT02365649.

Keywords: CELEST; European Quality of Life-5 Dimensions Visual Analog Scale; Inflammatory Bowel Disease Questionnaire; Janus kinase 1; Quality of life; Upadacitinib; Work Productivity and Activity Impairment Questionnaire

\section{Key Summary Points}

Why carry out this study?

Crohn's disease has a major impact on a patient's health-related quality of life, emphasizing the need for novel treatments.

We evaluated whether the Janus kinase 1 inhibitor upadacitinib improved quality of life and work productivity for patients with moderate to severe Crohn's disease based on data from a phase $2 b$ study.

\section{What was learned from this study?}

Statistically significant improvements from baseline were achieved as early as 8 weeks in quality of life and 16 weeks for work productivity and sustained for up to 52 weeks with upadacitinib in patients with moderate to severe Crohn's disease.

These phase 2 findings support the further assessment of safety, efficacy, quality of life, and work productivity outcomes with upadacitinib in phase 3 studies.

\section{DIGITAL FEATURES}

This article is published with digital features, including a summary slide, to facilitate understanding of the article. To view digital features for this article go to https://doi.org/10.6084/ m9.figshare.13720939.

\section{INTRODUCTION}

Crohn's disease (CD) is a chronic, inflammatory gastrointestinal disease with a prevalence of 318.5 cases per 100,000 persons in North America and 322.0 cases per 100,000 persons in Western Europe [1-5]. CD is characterized by chronic diarrhea, abdominal pain, and fatigue with disease progression leading to bowel damage and disability [1-4]. Consequently, this disease has a significant negative impact on a patient's health-related quality of life (HRQOL), including emotional and psychological issues, social and family interactions, and work-related difficulties [1, 6-8].

Current treatments for moderate to severe CD include corticosteroids, immunosuppressants, and biologic agents [2, 4]. However, issues with inadequate response, loss of response, and adverse events with these agents indicate the need for alternative medical treatments $[2,4,9,10]$. This is particularly significant for the improvement of patients' HRQOL and work productivity.

One relevant new target for $\mathrm{CD}$ is Janus kinase (JAK)-1, an important component of the signaling transduction pathway of cytokine receptors which play a major role in the pathogenesis of this disease [11-13]. Upadacitinib (ABT-494), an oral selective and reversible inhibitor of JAK-1 that more potently inhibits JAK-1 compared with JAK-2 and JAK-3, is currently being evaluated for the treatment of CD [14, 15]. Upadacitinib has recently been approved for adult patients with moderate to severe rheumatoid arthritis [16].

CELEST, a phase 2, placebo-controlled study of upadacitinib was completed in adult patients with moderate to severe $C D$ who had inadequate response or intolerance to immunosuppressants or tumor necrosis factor (TNF) antagonists [15]. Upadacitinib induced endoscopic remission in a significant proportion of patients compared with placebo at Week 12 or 16. Efficacy was maintained for most endpoints through Week 52, with adverse events consistent with those from previous studies for JAK inhibitors [15]. However, the impact of JAK inhibition on HRQOL and work productivity for 


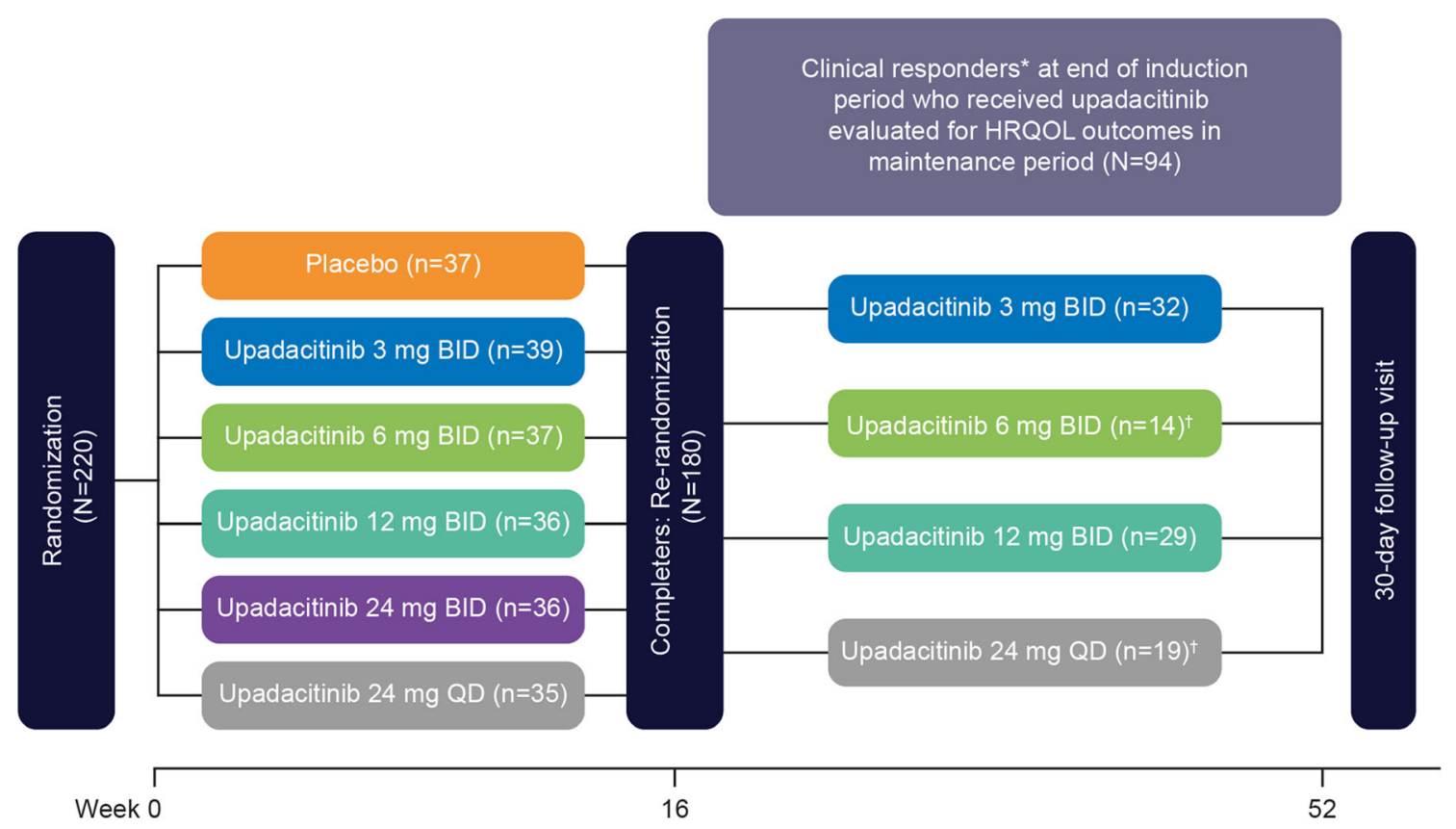

Fig. 1 Study design. ${ }^{*}$ Clinical responders defined as $\geq 30 \%$ decrease from baseline in average daily very soft/liquid stool frequency $\mathrm{OR} \geq 30 \%$ decrease from baseline in average daily abdominal pain score, and neither worse than baseline. ${ }^{\dagger}$ Upadacitinib 6-mg BID dosage

patients with $\mathrm{CD}$ is unknown. We now present the HRQOL and work productivity outcomes with upadacitinib for these patients at the end of the induction and maintenance periods from this phase 2 study.

\section{METHODS}

\section{Study Design}

CELEST was a phase 2, 52-week, multicenter, double-blind, randomized study consisting of a 16-week placebo-controlled, induction period followed by a 36-week maintenance period (Clinicaltrials.gov identifier: NCT02365649) (Fig. 1) [15]. For the induction period, patients were randomized in a $1: 1: 1: 1: 1: 1$ ratio to receive placebo or the immediate-release formulation of upadacitinib in oral doses of $3 \mathrm{mg}, 6 \mathrm{mg}$, $12 \mathrm{mg}$, or $24 \mathrm{mg}$ twice daily (BID) or $24 \mathrm{mg}$ once daily (QD). Patients who completed the 16-week induction period were re-randomized initiated and randomization for upadacitinib 24-mg QD dosage stopped with those currently enrolled at $24 \mathrm{mg}$ QD continuing treatment per protocol amendment. BID twice daily; $H R Q O L$ health-related quality of life; $Q D$ once daily

1:1:1 to receive double-blind maintenance therapy with the immediate-release formulation of upadacitinib at $3 \mathrm{mg}$ BID, $12 \mathrm{mg}$ BID, or $24 \mathrm{mg}$ QD for 36 weeks. A protocol amendment stopped the re-randomization into the $24-\mathrm{mg}$ QD arm in the maintenance period and initiated a 6-mg BID arm. Patients who were already assigned to $24 \mathrm{mg}$ QD continued to receive this dosage to the end of the study. All subsequent patients entering the maintenance period were re-randomized 1:1:1 to receive upadacitinib $3 \mathrm{mg}, 6 \mathrm{mg}$, or $12 \mathrm{mg}$ BID. Additional details have been previously reported [15].

This study was conducted in compliance with Good Clinical Practice guidelines, applicable local regulations, and the Declaration of Helsinki. This study protocol was approved by the relevant ethics committees or institutional review boards (see electronic supplementary material). All patients provided informed consent before study participation. 

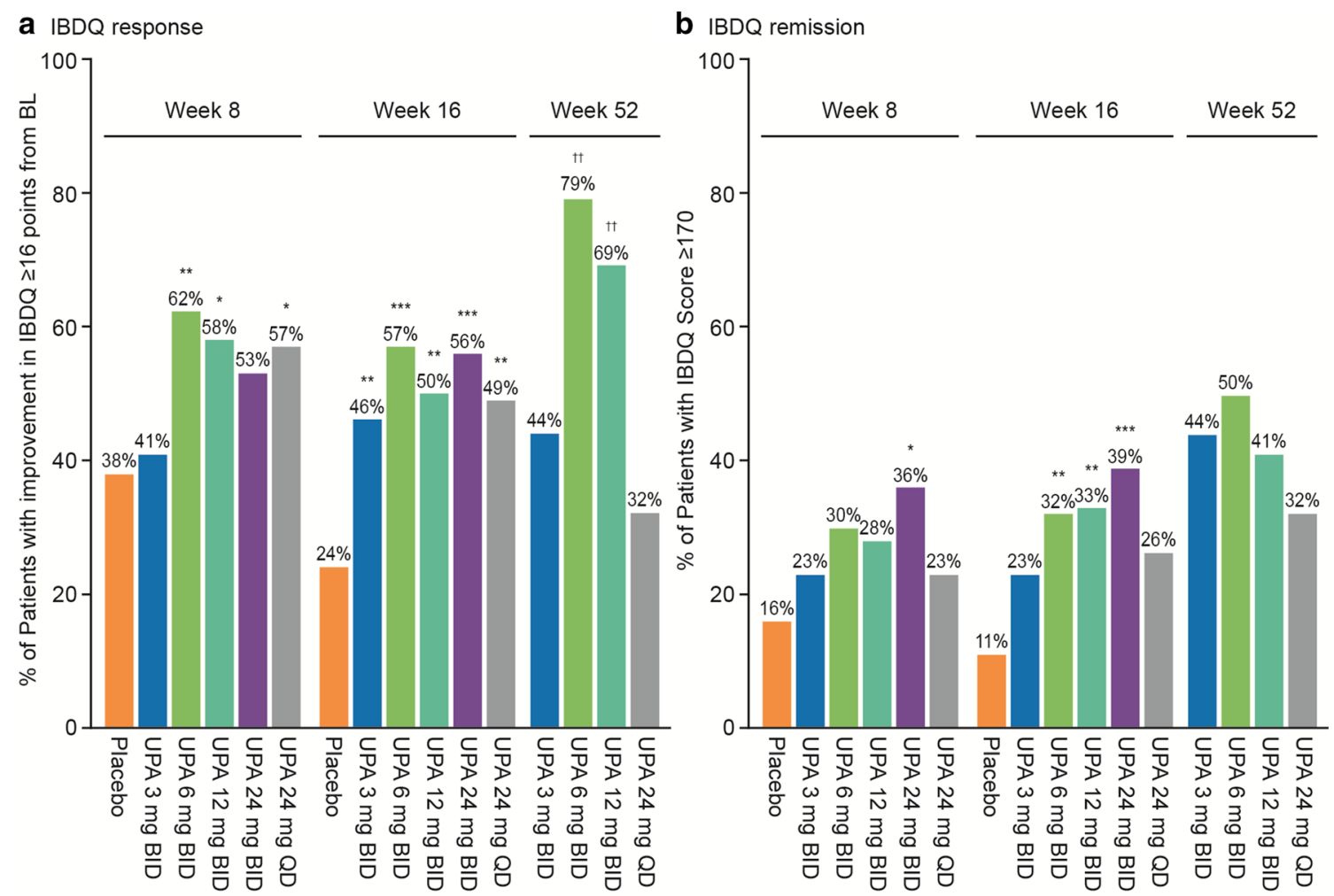

C Change in IBDQ total score

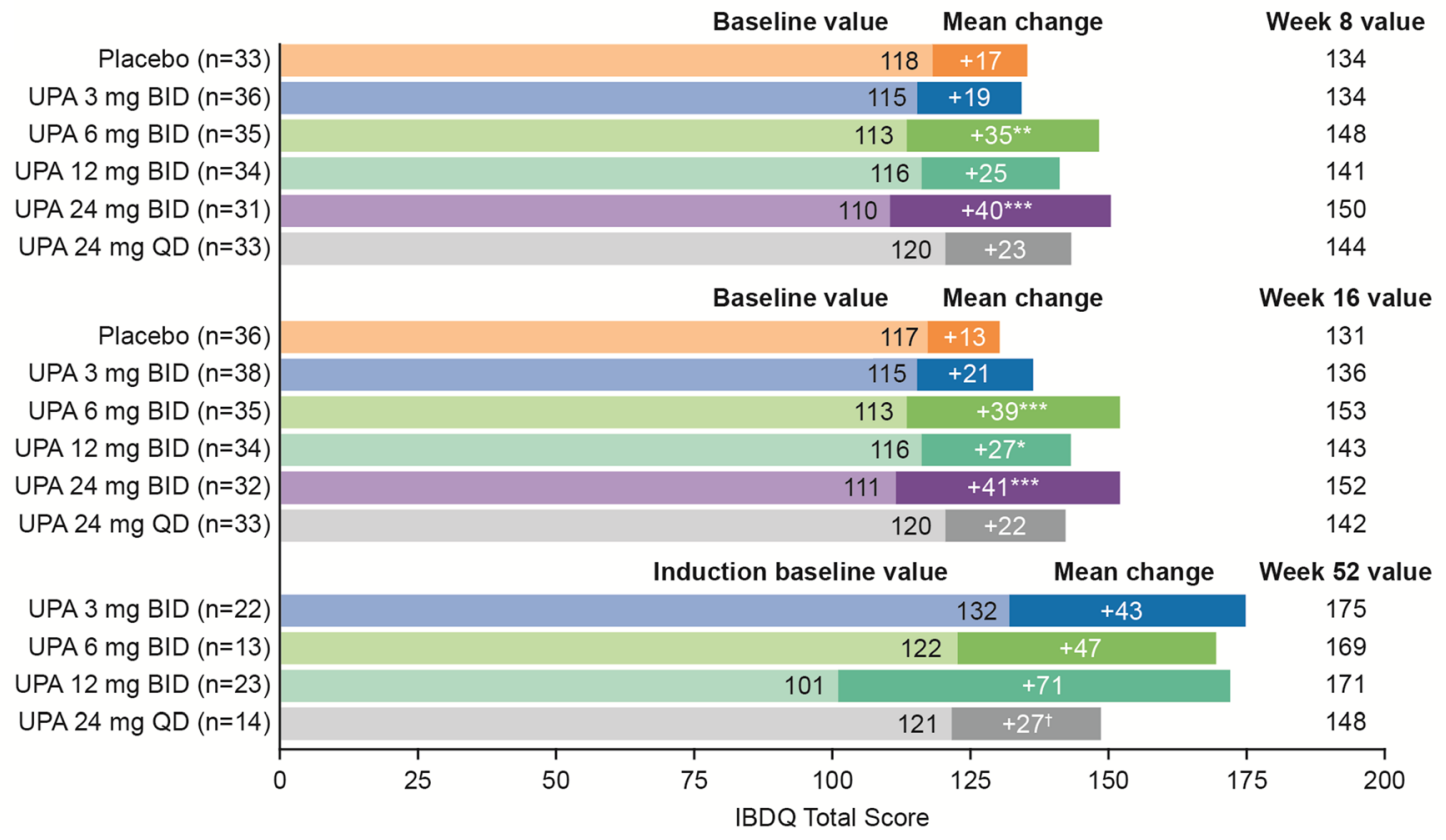


4Fig. 2 IBDQ improvements with upadacitinib. a IBDQ response. b IBDQ remission. c Change in IBDQ total score. ${ }^{*} P \leq 0.1$ vs. placebo. ${ }^{* *} P \leq 0.05$ vs. placebo. ${ }^{* * *} P \leq 0.01$ vs placebo. ${ }^{\dagger} P \leq 0.1$ vs UPA $3 \mathrm{mg}$ BID.

${ }^{\dagger} P \leq 0.05$ vs. UPA $3 \mathrm{mg}$ BID. IBDQ response and remission for induction period used the mITT population and LOCF, with $P$ values calculated based on CochranMantel-Haenszel test stratified by baseline SES-CD. IBDQ response and remission for maintenance period used the ITT population and OC, with period $P$ values calculated based on Chi-square test (or Fischer's exact test if $\geq 20 \%$ of the cells had expected cell count $<5$ ). Change in IBDQ score for induction period used the mITT population and LOCF, and was analyzed using ANCOVA, adjusting for treatment, baseline disease severity, and baseline value. Change in IBDQ score for maintenance period used the ITT population and OC, and was analyzed using ANCOVA, adjusting for treatment and baseline value. $A N C O V A$ analysis of covariance; $B I D$ twice daily; $B L$ baseline; $I B D Q$ Inflammatory Bowel Disease Questionnaire; ITT intent-to-treat; $L O C F$ last observation carried forward; mITT modified intent-totreat; $O C$ observed cases; $Q D$ once daily; SES-CD Simplified Endoscopic Score for Crohn's Disease; UPA upadacitinib

\section{Study Participants}

Patients $18-75$ years old with confirmed ileal, ileocolonic, or colonic CD for $\geq 3$ months, a CD Activity Index of 220-450, average daily liquid/ very soft stool frequency $\geq 2.5$ or daily abdominal pain score $\geq 2.0$, and Simplified Endoscopic Score for Crohn's Disease (SES-CD) $\geq 6$ (or $\geq 4$ for those with isolated ileal disease) were included in the study. Patients were also required to have an inadequate response/intolerance to at least one of the TNF antagonists approved for CD (adalimumab, infliximab, or certolizumab pegol) or (based on protocol amendment) to azathioprine, mercaptopurine, or methotrexate. Additional inclusion/exclusion criteria have been previously reported in the manuscript presenting the primary CELEST study results [15].

\section{Outcomes}

The study endpoints were to evaluate the improvements in HRQOL and work productivity with upadacitinib after Weeks 8,16 , and 52 of treatment compared with placebo (Weeks 8 and 16) or the 3-mg dose (Week 52). HRQOL was assessed in terms of the Inflammatory Bowel Disease Questionnaire (IBDQ) and European Quality of Life-5 Dimensions (EQ-5D) index and visual analog scale (VAS). Work productivity was assessed with the Work Productivity and Activity Impairment (WPAI) questionnaire. HRQOL and work productivity measures are reported for baseline, Week 8, Week 16, and Week 52 timepoints.

IBDQ is a disease-specific instrument that has been validated and commonly used for CD [17-21]. IBDQ is composed of 32 items graded on a 7-point Likert scale, which contains four dimensional score domains: bowel, emotional, social, and systemic [17-19]. Total score ranges from 32 to 224 , with higher scores indicating better HRQOL. Outcomes measured in this study were assessed at Weeks 8, 16, and 52: (1) percentage of patients with an increase in IBDQ score of $\geq 16$ points (minimum clinically important difference [22]) from baseline (IBDQ response); (2) percentage of patients with IBDQ score $\geq 170$ (IBDQ remission [23]); (3) mean change from baseline in IBDQ total score; (4) change in IBDQ domain scores from baseline to Week 16 and from Week 16 to Week 52; and (5) IBDQ score over the 52-week study period.

EQ-5D is a standardized, non-disease-specific instrument for describing and valuing qualityof-life consisting of five dimensions (index score): mobility, self-care, usual activities, pain/ discomfort, and anxiety/depression [24]. Each dimension has 5 levels: no problem, slight problem, moderate problem, severe problem, or unable to do the activity. EQ-5D also contains a VAS that ranges from 0 to 100 . Patients are asked to indicate the level that describes their current level of function or experience for each dimension. As a measure of health status, it provides a descriptive profile and can be used to generate a single index value for health status, where 0 is the worst health and 100 is the best health you can imagine. The outcome measured 
was mean change from baseline to Weeks 8 and 16 and from Week 16 to Week 52 in EQ-5D VAS score.

WPAI assesses the impact of the condition on work productivity losses and impairment in daily activity and is comprised of four domains: activity impairment, presenteeism (impairment while working), overall work impairment (productivity loss), and absenteeism (work time missed) [25-27]. Scores are expressed as impairment percentages, with higher numbers indicating greater impairment and less productivity. The outcome measured was mean change from baseline to Weeks 8 and 16 and from Week 16 to Week 52 in WPAI scores.

\section{Statistical Analyses}

All outcome measures were part of the primary analysis in the CELEST study. A full description of the statistical methods is reported in the primary manuscript of this study [15].

For the induction period, the modified intent-to-treat (mITT) analysis dataset was used for all the analyses. The mITT analysis dataset included randomized patients who took at least one dose of study drug during the induction period. Study outcomes were examined among treatment groups, with placebo as the reference group. The proportion of patients with IBDQ response and IBDQ remission, respectively, were analyzed based on the Cochran-Mantel-Haenszel test, adjusting for induction baseline SES-CD $(<15$ and $\geq 15)$. For IBDQ total and domain, EQ-5D index score and VAS, and WPAI scores, analysis of covariance (ANCOVA) was used to evaluate within-group changes from baseline to Weeks 8 and 16, adjusting for treatment, baseline disease severity, and baseline value. Missing data were reported using nonresponder imputation (NRI) and last observation carried forward (LOCF).

The intent-to-treat (ITT) analysis dataset included re-randomized patients in the maintenance period who were clinical responders (defined as $\geq 30 \%$ decrease from baseline in average daily very soft/liquid stool frequency $\mathrm{OR} \geq 30 \%$ decrease from baseline in average daily abdominal pain score, and neither worse than baseline) in the induction period. Study outcomes were examined among treatment groups, with upadacitinib $3 \mathrm{mg}$ BID as the reference group. The proportion of patients with IBDQ response and IBDQ remission were analyzed based on the chi-square test (or Fischer's exact test if $\geq 20 \%$ of the cells had expected cell count $<5$ ). Mean change in IBDQ, EQ-5D VAS, and WPAI from Week 16 to Week 52 were analyzed using ANCOVA, adjusting for treatment and induction baseline value. Missing data were reported using NRI and observed cases.

All tests of significance were at the 2-sided $10 \%$ level of significance $(P \leq 0.1)$, as used in the previously reported primary study [15], and which is commonly used in phase $2 b$ trials associated with dose selection for future phase 3 studies [28]. All reported $P$ values are nominal. All data analyses were performed using SAS v.9.4 (SAS Institute, Cary, NC, USA).

\section{RESULTS}

\section{Study Population}

A total of 220 patients with moderately to severely active CD were randomized to induction treatment, with 180 completing the induction phase and re-randomized to upadacitinib for the maintenance period (Fig. 1). Of these 180 patients, 153 received upadacitinib during induction of whom 94 patients were clinical responders. Maintenance phase results are provided for the 94 clinical responders.

At baseline, median disease duration was 9.6 years, with a high proportion (96\%) having prior TNF antagonist exposure and 64\% exposed to two or more biologics (Table 1) [15]. Baseline IBDQ scores, EQ-5D index score and VAS values, and WPAI domain scores were numerically similar between treatment groups (Table 1).

\section{IBDQ Changes with Upadacitinib}

At Week 16, a significantly higher percentage of patients had a clinically meaningful IBDQ 
Table 1 Patient demographics and baseline characteristics (mITT population)

\begin{tabular}{|c|c|c|c|c|c|c|}
\hline Variable & $\begin{array}{l}\text { Placebo } \\
(n=37)\end{array}$ & $\begin{array}{l}\text { Upadacitinib } \\
3 \mathrm{mg} \text { BID }(n=39)\end{array}$ & $\begin{array}{l}\text { Upadacitinib } \\
6 \mathrm{mg} \text { BID }(n=37)\end{array}$ & $\begin{array}{l}\text { Upadacitinib } \\
12 \mathrm{mg} \text { BID } \\
(n=36)\end{array}$ & $\begin{array}{l}\text { Upadacitinib } \\
24 \mathrm{mg} \text { BID } \\
(n=36)\end{array}$ & $\begin{array}{l}\text { Upadacitinib } \\
24 \mathrm{mg} Q D \\
(n=35)\end{array}$ \\
\hline Age, years, median (range) & $40(20-68)$ & $37(19-66)$ & $39(22-76)$ & $41(19-70)$ & $44(20-65)$ & $41(21-64)$ \\
\hline Female, $n(\%)$ & $24(64.9)$ & $19(48.7)$ & $21(56.8)$ & $17(47.2)$ & $25(69.4)$ & $19(54.3)$ \\
\hline Disease duration, years, median (range) & $8.7(1.2-41.6)$ & $10.7(0.1-44.7)$ & $8.8(2.2-46.4)$ & $9.1(1.2-38.3)$ & $14.1(1.1-35.5)$ & $10.8(0.7-36.3)$ \\
\hline CDAI, median (range) & $276(188-447)$ & $288(180-445)$ & $296(230-599)$ & $280(224-446)$ & $278(162-556)$ & $305(231-421)$ \\
\hline \multicolumn{7}{|l|}{ IBDQ } \\
\hline Mean \pm SD & $118.0 \pm 28.5$ & $115.2 \pm 27.5$ & $113.7 \pm 25.9$ & $115.2 \pm 36.1$ & $113.8 \pm 36.0$ & $120.7 \pm 36.3$ \\
\hline Missing data & 0 & 1 & 1 & 1 & 0 & 1 \\
\hline \multicolumn{7}{|l|}{ EQ-5D index score } \\
\hline Mean \pm SD & $0.65 \pm 0.24$ & $0.69 \pm 0.17$ & $0.71 \pm 0.18$ & $0.67 \pm 0.21$ & $0.69 \pm 0.20$ & $0.68 \pm 0.22$ \\
\hline Missing data & 0 & 0 & 0 & 1 & 0 & 1 \\
\hline \multicolumn{7}{|l|}{ EQ-5D VAS } \\
\hline Mean \pm SD & $48.6 \pm 14.6$ & $49.4 \pm 18.7$ & $47.8 \pm 16.4$ & $50.7 \pm 17.6$ & $51.6 \pm 18.5$ & $50.7 \pm 17.0$ \\
\hline Missing data & 0 & 0 & 0 & 2 & 0 & 2 \\
\hline \multicolumn{7}{|l|}{ WPAI: \% activity impairment } \\
\hline Mean $\pm S D$ & $60.3 \pm 26.5$ & $63.3 \pm 19.8$ & $61.9 \pm 18.2$ & $62.6 \pm 24.2$ & $57.5 \pm 23.5$ & $60.6 \pm 24.1$ \\
\hline Available data $^{\mathrm{a}}$ & 37 & 39 & 36 & 35 & 36 & 33 \\
\hline \multicolumn{7}{|l|}{ WPAI: \% presenteeism } \\
\hline Mean \pm SD & $41.8 \pm 23.2$ & $58.8 \pm 26.4$ & $57.2 \pm 23.2$ & $47.3 \pm 27.9$ & $47.5 \pm 25.5$ & $47.0 \pm 26.5$ \\
\hline Available data ${ }^{a}$ & 22 & 17 & 25 & 15 & 20 & 23 \\
\hline \multicolumn{7}{|l|}{ WPAI: \% overall work impairment } \\
\hline Mean $\pm S D$ & $52.1 \pm 29.1$ & $66.5 \pm 27.4$ & $61.4 \pm 25.0$ & $57.1 \pm 29.3$ & $52.4 \pm 29.8$ & $53.3 \pm 30.7$ \\
\hline Available data ${ }^{a}$ & 23 & 19 & 24 & 15 & 20 & 24 \\
\hline \multicolumn{7}{|l|}{ WPAI: \% absenteeism } \\
\hline Mean $\pm S D$ & $19.8 \pm 29.5$ & $24.0 \pm 36.0$ & $15.6 \pm 26.2$ & $23.4 \pm 40.2$ & $15.2 \pm 26.5$ & $20.7 \pm 31.4$ \\
\hline Available data ${ }^{a}$ & 23 & 19 & 24 & 15 & 20 & 24 \\
\hline \multicolumn{7}{|l|}{ Prior anti-TNF agents, $n$ (\%)[15] } \\
\hline 0 & $2(5.4)$ & $2(5.1)$ & $1(2.7)$ & $2(5.6)$ & 0 & $2(5.7)$ \\
\hline 1 & $15(40.5)$ & $17(43.6)$ & $12(32.4)$ & $6(16.7)$ & $10(27.8)$ & $10(28.6)$ \\
\hline 2 & $15(40.5)$ & $16(41.0)$ & $20(54.1)$ & $24(66.7)$ & $15(41.7)$ & $16(45.7)$ \\
\hline$\geq 3$ & $5(13.5)$ & $4(10.3)$ & $4(10.8)$ & $4(11.1)$ & $11(30.6)$ & $7(20.0)$ \\
\hline
\end{tabular}

a Available data for all patients that are employed

$B I D$ twice daily, $C D A I$ Crohn's Disease Activity Index, EQ-5D European Quality of Life-5 Dimensions, IBDQ Inflammatory Bowel Disease Questionnaire, $m I T T$ modified intent-to-treat, $Q D$ once daily, $S D$ standard deviation, $T N F$ tumor necrosis factor, $V A S$ visual analog scale, $W P A I$ Work Productivity and Activity Impairment questionnaire

response with upadacitinib compared with placebo for all dosages, with the upadacitinib 6-mg and 24-mg BID groups having the most significant increases $(P \leq 0.01)$ (Fig. 2a). Greater percentages of patients with a clinically meaningful IBDQ response were also observed at Week 8 with upadacitinib $6 \mathrm{mg}$ BID, $12 \mathrm{mg}$ BID, and $24 \mathrm{mg}$ QD relative to placebo $(P \leq 0.1)$ and at Week 52 with upadacitinib $6 \mathrm{mg}$ BID and $12 \mathrm{mg}$ BID relative to the $3-\mathrm{mg}$ dosage $(P \leq 0.05)$.

A significantly greater percentage of patients also achieved IBDQ remission at Week 16 with upadacitinib versus placebo for the 6-mg, 12-mg, and 24-mg BID dosages $(P \leq 0.05)$ (Fig. 2b), with higher percentages of patients 
achieving IBDQ remission at Week 8 with upadacitinib versus placebo, particularly for the 24-mg BID dose $(P \leq 0.1)$. At Week 52 of the maintenance period, a numerically higher percentage of patients achieved IBDQ remission with all dosages of upadacitinib compared with Week 16, although no significant improvements were observed versus the 3-mg dosage.

Significant mean increases from baseline in IBDQ total score were obtained at Weeks 8 and 16 with both the upadacitinib 6-mg and 24-mg BID dosages compared with placebo $(P \leq 0.05)$ (Fig. 2c; see Table S1 in the electronic supplementary material). IBDQ total scores rose further in the maintenance period with upadacitinib treatment for all treatment groups (Fig. 2c; see Table S2 in the electronic supplementary material).

Improvements in the individual IBDQ domain scores (bowel, social, emotional, and systemic) with upadacitinib were generally consistent with the total IBDQ score changes, with patients receiving upadacitinib $6 \mathrm{mg}$ BID and $24 \mathrm{mg}$ BID having the most significant mean changes in the induction period $(P \leq 0.05)$. In the maintenance period, the highest mean change from induction baseline was achieved with upadacitinib $12 \mathrm{mg}$ BID compared with the other treatment groups for the individual IBDQ domain scores (see Fig. S1 in the electronic supplementary material).

\section{EQ-5D Changes with Upadacitinib}

Similar to IBDQ, upadacitinib $6 \mathrm{mg}$ BID and $24 \mathrm{mg}$ BID had the most significant mean EQ-5D VAS score increases from baseline compared with the other treatment groups in the induction period at both Weeks 8 and 16 $(P$ values of 0.014 and 0.011 at Week 16, respectively, vs. placebo) (Fig. 3 ; see Table S1 in the electronic supplementary material). In the maintenance period, the upadacitinib 12-mg BID group had significant improvements from induction baseline in EQ-5D VAS score versus upadacitinib $3 \mathrm{mg}$ BID $(P=0.052)$ (Fig. 3; see Table S2 in the electronic supplementary material).
The greatest changes from baseline in the EQ-5D index scores versus placebo were with the 12-mg BID $(P \leq 0.1)$ and 24-mg BID $(P \leq 0.01)$ dosages at Week 8 and with the $3-\mathrm{mg}$ BID $(P \leq 0.1)$, 6-mg BID $(P \leq 0.05), 24-\mathrm{mg}$ BID $(P \leq 0.01)$, and $24-\mathrm{mg}$ QD $(P \leq 0.1)$ dosages at Week 16 (see Fig. S2 and Table S1 in the electronic supplementary material). In the maintenance period, numerical improvements were observed with the upadacitinib 12-mg BID group from induction baseline in EQ-5D index score versus upadacitinib $3 \mathrm{mg}$ BID (see Fig. S2 and Table S2 in the electronic supplementary material).

\section{WPAI Changes with Upadacitinib}

Decreases of at least 7\% in each of the WPAI domains are regarded as sizable changes and minimally important differences [29]. Compared with placebo, clinically meaningful improvements of more than $7 \%$ in activity impairment were observed with the 6-mg BID $(P \leq 0.05)$ and 24 -mg BID $(P \leq 0.01)$ dosages; and in overall work impairment with $24 \mathrm{mg}$ BID $(P \leq 0.1)$ at Week 16 (Fig. 4; see Table S1 in the electronic supplementary material). Although improvements in WPAI were numerically greater at Week 52 of the maintenance period compared with Week 16 for all upadacitinib dosages, no significant increases versus the 3-mg BID dosage were observed (Fig. 4; see Table S2 in the electronic supplementary material).

\section{DISCUSSION}

In this placebo-controlled trial, patients with moderately to severely active CD had significant improvements in HRQOL and work productivity outcomes after a 16-week induction treatment with upadacitinib which were sustained in a 36-week maintenance period. In particular, significant improvements with upadacitinib in HRQOL were achieved after 16 weeks of treatment in IBDQ with the 6-, 12-, and 24-mg BID dosages and in EQ-5D VAS and activity impairment with the 6- and 24-mg BID dosages versus placebo. For the 6- and 24-mg BID dosages, 


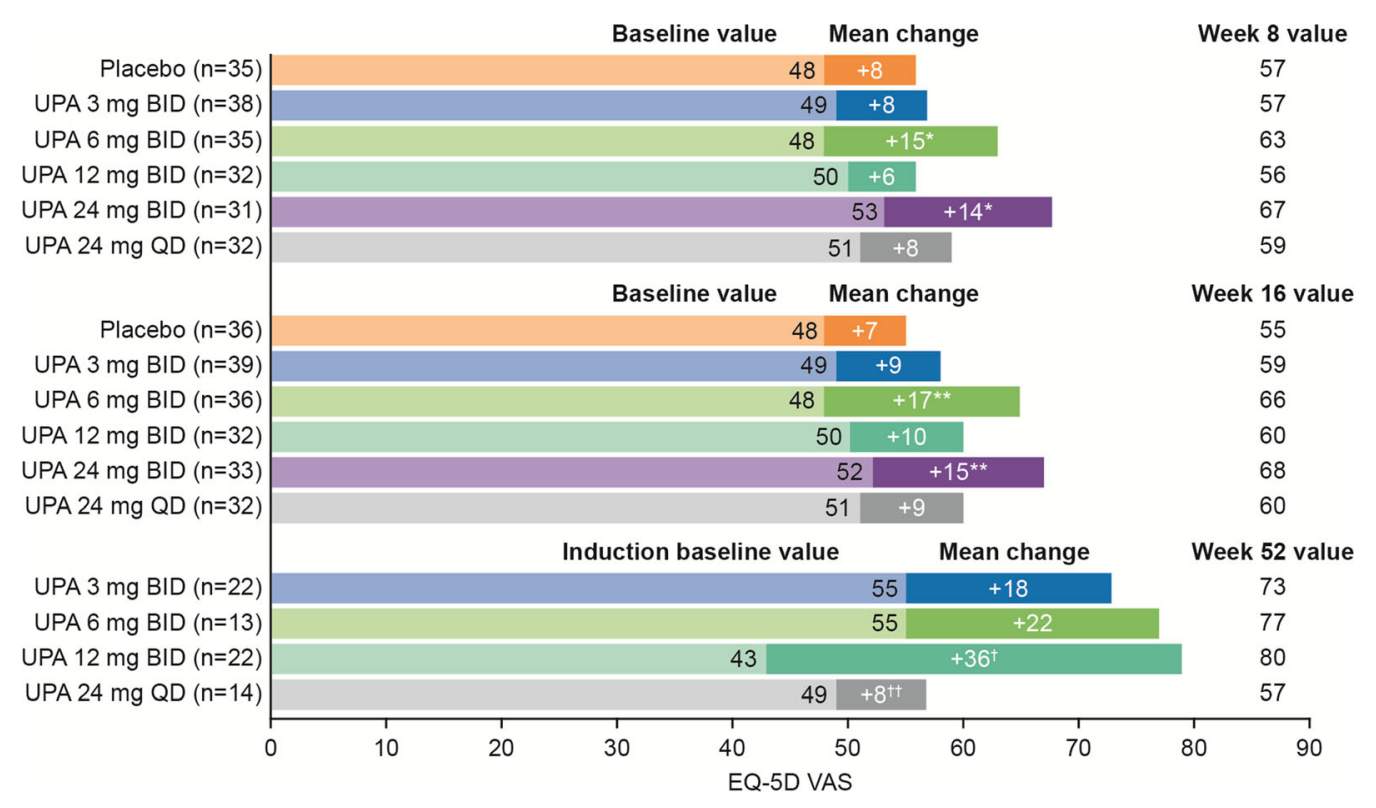

Fig. 3 Change in EQ-5D VAS score with upadacitinib. ${ }^{*} P \leq 0.1$ vs. placebo. ${ }^{* *} P \leq 0.05$ vs. placebo. ${ }^{\dagger} P \leq 0.1$ vs. UPA $3 \mathrm{mg}$ BID. ${ }^{\dagger \dagger} P \leq 0.05$ vs. UPA $3 \mathrm{mg}$ BID. Change in EQ-5D VAS score for induction period used the mITT population and LOCF, and was analyzed using ANCOVA, adjusting for treatment, baseline disease severity, and baseline value. Change in EQ-5D VAS score

these improvements were observed as early as Week 8 for mean change from baseline in IBDQ and EQ-5D VAS scores compared with placebo. Further improvements were observed after 1 year of treatment, with $79 \%$ and $69 \%$ of patients achieving IBDQ response with the 6- and 12-mg BID dosages.

The results observed with the BID dose regimen were consistent with what was reported for the primary clinical and endoscopic efficacy results [15]. The reduced HRQOL improvements with the QD dose regimen during the maintenance period may potentially be because of its modest pharmacokinetic properties, as previously reported [15].

To the best of our knowledge, this is the first report on the effects of a JAK inhibitor on HRQOL and work productivity for CD. HRQOL and work productivity outcomes that were positively affected by upadacitinib in this study included symptoms and functioning, which are considered highly important to patients with CD. This includes outcomes related to bowel for maintenance period used the ITT population and OC, and was analyzed using ANCOVA, adjusting for treatment and baseline value. ANCOVA analysis of covariance, BID twice daily, EQ-5D VAS European Quality of Life-5 Dimensions visual analog scale, ITT intent-to-treat, $L O C F$ last observation carried forward, $m I T T$ modified intent-totreat, $O C$ observed cases, $Q D$ once daily, UPA upadacitinib

symptoms (e.g., frequent and loose bowel movements and abdominal cramping), systemic symptoms (e.g., fatigue and general feeling of being unwell), emotional functioning (e.g., feelings of frustration and depression), social impairment (e.g., avoiding activities where no restrooms are near and cancelling social events), and performing daily activities (e.g., housework, childcare, shopping, exercising, and studying) $[18,27,30,31]$. Thus, the improvements with upadacitinib noted in this study could have a major impact on multiple aspects of a patient's quality of life.

These HRQOL and work productivity results are consistent with the positive findings that were recently reported for the primary and key secondary endpoints from the CELEST study program for upadacitinib [15]. Additionally, these findings are in line with reports for biologics (e.g., TNF, interleukin-12/23, and integrin receptor antagonists) that patients who have clinical responses to these agents have HRQOL and work productivity benefits [32-37]. 


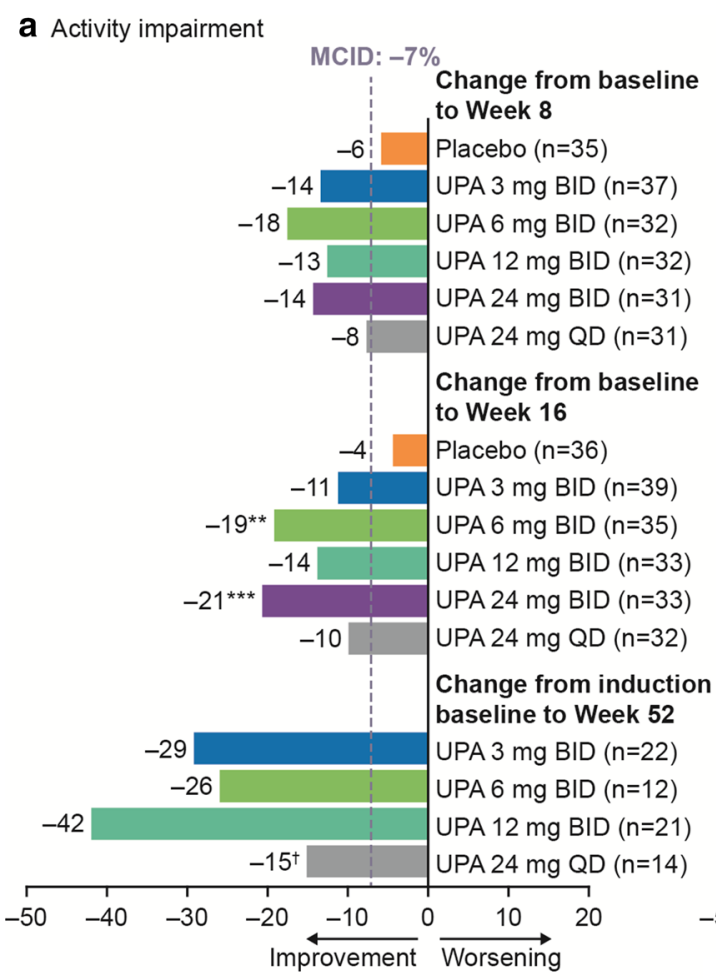

C Overall work impairment

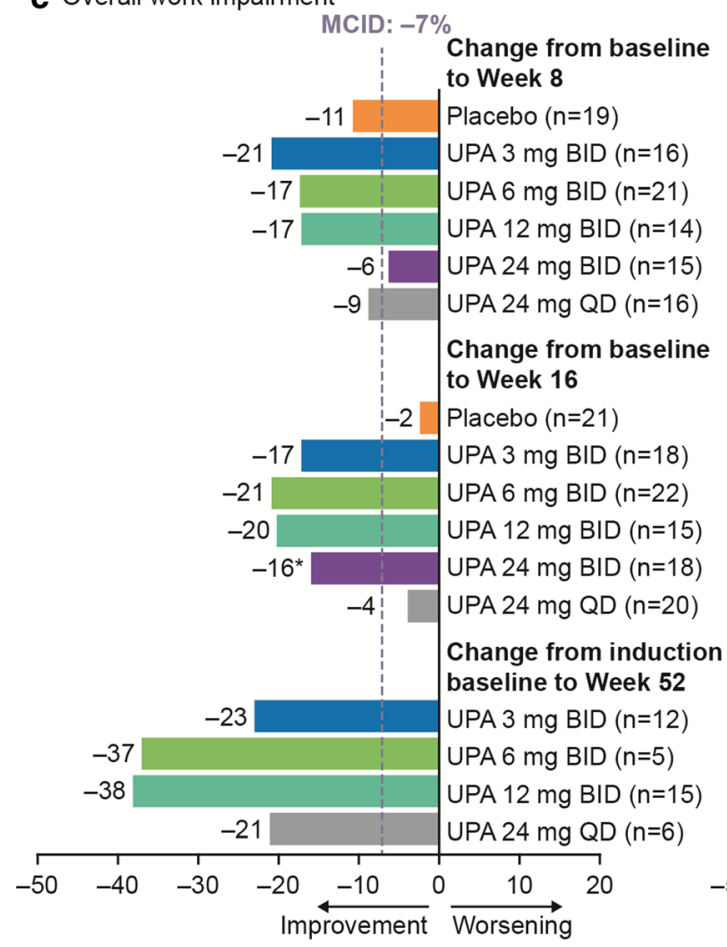

b Presenteeism

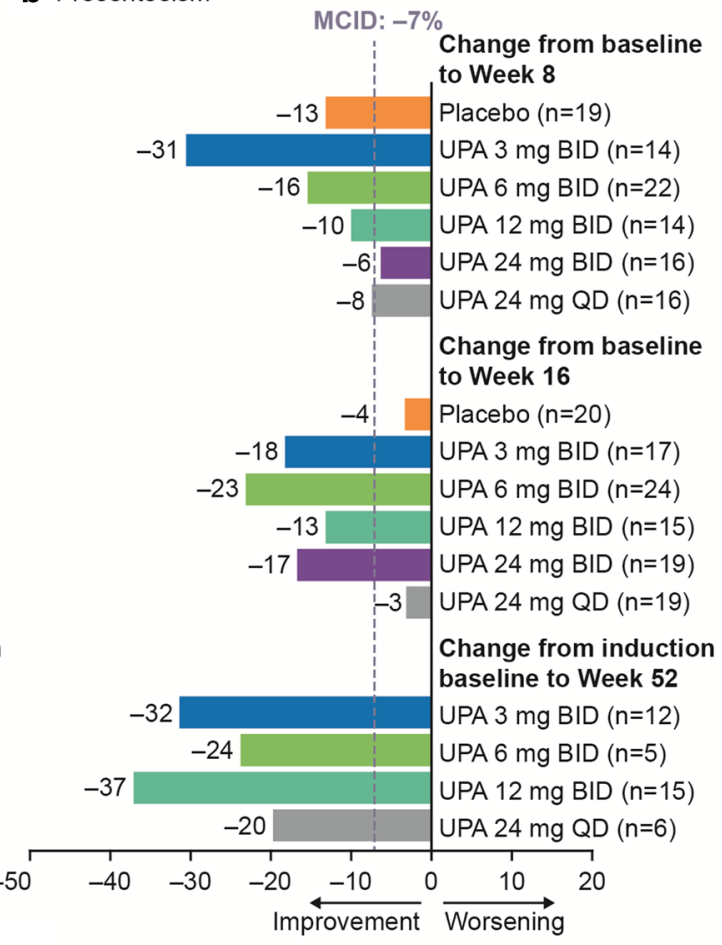

d Absenteeism

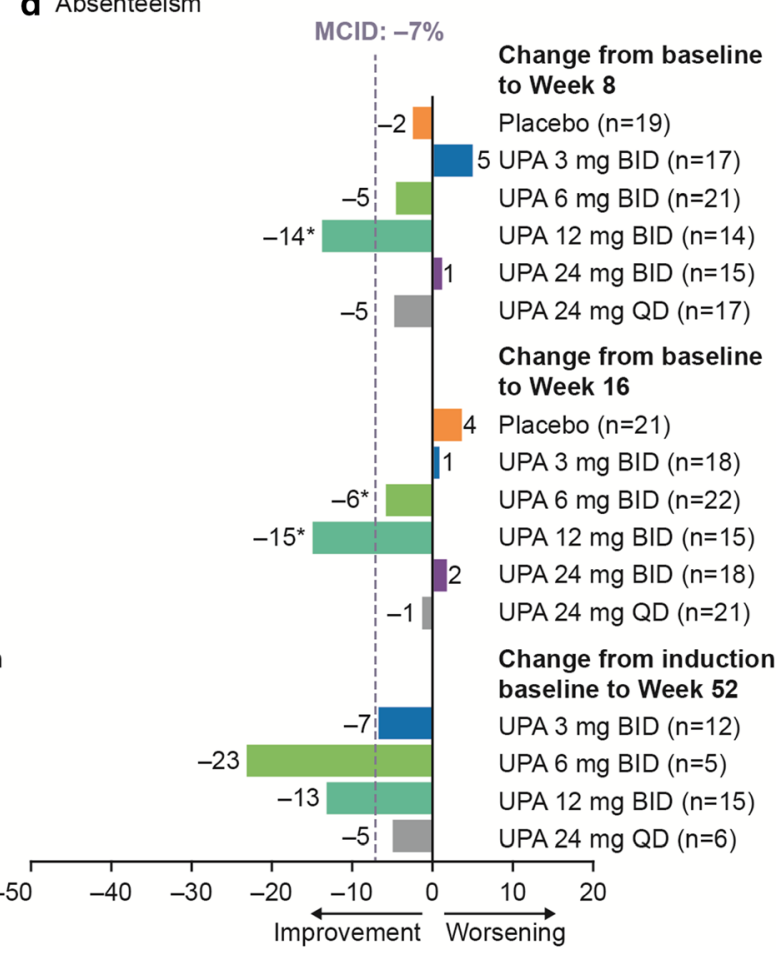


4 Fig. 4 Mean change in WPAI score with upadacitinib. a Activity impairment. b Presenteeism. c Overall work impairment. d Absenteeism. ${ }^{*} P \leq 0.1$ vs. placebo. ${ }^{* *} P \leq 0.05$ vs. placebo. ${ }^{* * *} P \leq 0.01$ vs. placebo. ${ }^{\dagger} P \leq 0.1$ vs. UPA $3 \mathrm{mg}$ BID. Change in WPAI score for induction period used the mITT population and LOCF, and was analyzed using ANCOVA, adjusting for treatment, baseline disease severity, and baseline value. Change in WPAI score for maintenance period used the ITT population and OC, and was analyzed using ANCOVA, adjusting for treatment and baseline value. ANCOVA analysis of covariance; BID twice daily; ITT intent-to-treat; LOCF last observation carried forward; MCID minimum clinically important difference; mITT modified intent-totreat; OC observed cases; QD once daily; UPA upadacitinib; WPAI Work Productivity and Activity Impairment questionnaire

There is a considerable need for novel treatments for CD, particularly as current therapeutic options, including corticosteroids, immunomodulatory agents such as azathioprine, 6-mercaptopurine, or methotrexate, and biologics, such as TNF antagonists, are ineffective in a relevant percentage of patients and can be associated with adverse effects that can limit their use $[2,4,9,10]$. The CELEST study enrolled patients with longstanding and refractory $\mathrm{CD}$, and yet clinical remission was achieved by up to $27 \%$ of patients at Week 16 with upadacitinib (6 mg BD vs. 11\% for placebo; $P \leq 0.1$ ), and endoscopic remission was obtained by up to $22 \%$ of patients at Week 12 or 16 (24 mg BID, none for placebo, $P \leq 0.01$ ) [15]. Safety findings were consistent with those from previous studies of JAK inhibitors [15]. Results from clinical, endoscopic, HRQOL, and work productivity endpoints in the CELEST study together indicate that there is a potential for JAK-1 inhibitors to be an additional option for the treatment of moderate to severe $C D$. As this is a dose-finding study with a limited number of patients per treatment group, and no clear dose-response across the HRQOL measures, a more robust phase 3 trial is ongoing to confirm these findings.

Another limitation of this study was the lack of a placebo control during the maintenance phase, in which the use of upadacitinib $3 \mathrm{mg}$ BID as a comparator could have resulted in an underestimation of the improvements observed with the other upadacitinib dosages. Therefore, conclusions regarding the maintenance of effects observed in the induction phase need to be viewed with caution. Furthermore, with all maintenance studies including only responders, care must be taken when interpreting effect size and extrapolating results to all patients.

\section{CONCLUSIONS}

This is the first study to evaluate the impact of JAK inhibition on HRQOL and work productivity in patients with $\mathrm{CD}$. We demonstrated that 16 weeks of induction therapy with upadacitinib resulted in significant improvements in HRQOL and work productivity in patients with moderately to severely active CD that were sustained with an additional 36 weeks of treatment. A phase 3 trial is currently being initiated to confirm safety, efficacy, and HRQOL and work productivity outcomes with upadacitinib from the phase 2 study in patients with moderately to severely active CD.

\section{ACKNOWLEDGEMENTS}

AbbVie and the authors thank the patients who participated in the study and all of the study investigators for their contributions.

Funding. Sponsorship for this study and the journal's Rapid Service fee and Open Access charge was funded by AbbVie Inc., North Chicago, IL.

Medical Writing Assistance. Medical writing assistance was provided by Alan Saltzman, PhD, CMPP, of Fishawack Facilitate Ltd, part of Fishawack Health, and was funded by AbbVie Inc., North Chicago, IL.

Authorship. All named authors meet the International Committee of Medical Journal Editors (ICMJE) criteria for authorship for this article, take responsibility for the integrity of the work as a whole, and have given their approval for this version to be published. AbbVie sponsored the study; contributed to the 
design; participated in collection, analysis, and interpretation of data; and in writing, reviewing and approval of the final version.

Disclosures. L. Peyrin-Biroulet reports personal fees from Galapagos, AbbVie, Janssen, Genentech, Ferring, Tillots, Pharmacosmos, Celltrion, Takeda, Boehringer Ingelheim, Pfizer, Index Pharmaceuticals, Sandoz, Celgene, Biogen, Samsung Bioepis, Alma, Sterna, Nestle, Inotrem, Enterome, Allergan, MSD, Roche, Arena, Gilead, Hikma, Amgen, BMS, Vifor, Norgine, Mylan, Lilly, Fresenius Kabi, Oppilan Pharma, Sublimity Therapeutics, Applied Molecular Transport, OSE Immunotherapeutics, Enthera, and Theravance; has received grants from AbbVie, MSD, and Takeda; and has stock options from CTMA. E. Louis has received research grants from Takeda, Pfizer, and Janssen; has received educational grants from AbbVie, MSD, Takeda, and Janssen; reports speaker fees from AbbVie, Ferring, MSD, Falk, Takeda, Hospira, Janssen, and Pfizer; participated in advisory boards for AbbVie, Ferring, MSD, Takeda, Celgene, Hospira, Janssen, and Pfizer; and is a consultant for AbbVie. E. V. Loftus has received consulting fees from AbbVie, Takeda, Janssen, UCB, Amgen, Pfizer, Eli Lilly, Boehringer Ingelheim, Bristol Myers Squibb, Celgene, Gilead, Genentech, and Celltrion Healthcare and research support from AbbVie, Takeda, Janssen, UCB, Amgen, Pfizer, Genentech, Receptos, Gilead, Celgene, Bristol Myers Squibb, Theravance, and Robarts Clinical Trials. A. Lacerda, Q. Zhou, and Y. Sanchez Gonzalez are AbbVie employees and may own AbbVie stock/stock options. S. Ghosh is a steering committee member for Pfizer, Janssen, AbbVie, BMS, Celgene, and Boehringer-Ingelheim and has received speaker honoraria from AbbVie, Janssen, Takeda, Shield, Ferring, and Falk Pharma.

Compliance with Ethics Guidelines. This study was conducted in compliance with Good Clinical Practice guidelines, applicable local regulations, and the Declaration of Helsinki. This study protocol was approved by the relevant ethics committees or institutional review boards (see electronic supplementary material).
All patients provided informed consent before study participation.

Data Availability. AbbVie is committed to responsible data sharing regarding the clinical trials we sponsor. This includes access to anonymized, individual, and trial-level data (analysis data sets), as well as other information (e.g., protocols and clinical study reports), as long as the trials are not part of an ongoing or planned regulatory submission. This includes requests for clinical trial data for unlicensed products and indications. These clinical trial data can be requested by any qualified researchers who engage in rigorous, independent scientific research and will be provided after review and approval of a research proposal and statistical analysis plan and execution of a data sharing agreement. Data requests can be submitted at any time, and the data will be accessible for 12 months, with possible extensions considered. For more information on the process, or to submit a request, visit https://www.abbvie.com/ our-science/clinical-trials/clinical-trialsdata-andinformation-sharing/data-and-informationsharing-with-qualifiedresearchers.html.

Open Access. This article is licensed under a Creative Commons Attribution-NonCommercial 4.0 International License, which permits any non-commercial use, sharing, adaptation, distribution and reproduction in any medium or format, as long as you give appropriate credit to the original author(s) and the source, provide a link to the Creative Commons licence, and indicate if changes were made. The images or other third party material in this article are included in the article's Creative Commons licence, unless indicated otherwise in a credit line to the material. If material is not included in the article's Creative Commons licence and your intended use is not permitted by statutory regulation or exceeds the permitted use, you will need to obtain permission directly from the copyright holder. To view a copy of this licence, visit http://creativecommons.org/licenses/by$\mathrm{nc} / 4.0 /$. 


\section{REFERENCES}

1. Roda G, Chien Ng S, Kotze PG, et al. Crohn's disease. Nat Rev Dis Prim. 2020;6:22.

2. Lichtenstein GR, Loftus EV, Isaacs KL, Regueiro MD, Gerson LB, Sands BE. ACG clinical guideline: management of Crohn's disease in adults. Am J Gastroenterol. 2018;113:481-517.

3. Torres J, Mehandru S, Colombel JF, Peyrin-Biroulet L. Crohn's disease. Lancet. 2017;389:1741-55.

4. Gomollon F, Dignass A, Annese V, et al. 3rd European evidence-based consensus on the diagnosis and management of Crohn's disease 2016: part 1: diagnosis and medical management. J Crohns Colitis. 2017;11:3-25.

5. Ng SC, Shi HY, Hamidi N, et al. Worldwide incidence and prevalence of inflammatory bowel disease in the 21st century: a systematic review of population-based studies. Lancet. 2018;390: 2769-78.

6. Wilburn J, McKenna SP, Twiss J, Kemp K, Campbell S. Assessing quality of life in Crohn's disease: development and validation of the Crohn's Life Impact Questionnaire (CLIQ). Qual Life Res. 2015;24:2279-88.

7. Vergara M, Montserrat A, Casellas F, et al. A new validation of the Spanish Work Productivity and Activity Impairment Questionnaire-Crohn's disease version. Value Health. 2011;14:859-61.

8. Gower-Rousseau C, Sarter H, Savoye G, et al. Validation of the inflammatory bowel disease disability index in a population-based cohort. Gut. 2017;66: 588-96.

9. Ben-Horin S, Chowers Y. Review article: loss of response to anti-TNF treatments in Crohn's disease. Aliment Pharmacol Ther. 2011;33:987-95.

10. Billioud V, Sandborn WJ, Peyrin-Biroulet L. Loss of response and need for adalimumab dose intensification in Crohn's disease: a systematic review. Am J Gastroenterol. 2011;106:674-84.

11. Hammaren HM, Virtanen AT, Raivola J, Silvennoinen $\mathrm{O}$. The regulation of JAKs in cytokine signaling and its breakdown in disease. Cytokine. 2019;118:48-63.

12. Yamamoto-Furusho JK. Inflammatory bowel disease therapy: blockade of cytokines and cytokine signaling pathways. Curr Opin Gastroenterol. 2018;34:187-93.
13. Cui D, Huang G, Yang D, Huang B, An B. Efficacy and safety of interferon-gamma-targeted therapy in Crohn's disease: a systematic review and metaanalysis of randomized controlled trials. Clin Res Hepatol Gastroenterol. 2013;37:507-13.

14. Parmentier JM, Voss J, Graff C, et al. In vitro and in vivo characterization of the JAK1 selectivity of upadacitinib (ABT-494). BMC Rheumatol. 2018;2: 23.

15. Sandborn WJ, Feagan BG, Loftus EV Jr, et al. Efficacy and safety of upadacitinib in a randomized trial of patients with Crohn's disease. Gastroenterology. 2020;158:2123-38.

16. RINVOQ ${ }^{\mathrm{TM}}$ (upadacitinib) prescribing information, AbbVie Inc., North Chicago, IL, USA. 2019.

17. Irvine EJ, Feagan B, Rochon J, et al. Quality of life: a valid and reliable measure of therapeutic efficacy in the treatment of inflammatory bowel disease. Canadian Crohn's Relapse Prevention Trial Study Group. Gastroenterology. 1994;106:287-96.

18. Mitchell A, Guyatt G, Singer J, et al. Quality of life in patients with inflammatory bowel disease. J Clin Gastroenterol. 1988;10:306-10.

19. Guyatt G, Mitchell A, Irvine EJ, et al. A new measure of health status for clinical trials in inflammatory bowel disease. Gastroenterology. 1989;96:804-10.

20. Irvine EJ, Feagan B, Rochon J, et al. Quality of life: a valid and reliable measure of therapeutic efficacy in the treatment of inflammatory bowel disease. Canadian Crohn's Relapse Prevention Trial Study Group. Gastroenterology. 1994;106:287-96.

21. Abbass M, Cepek J, Parker CE, et al. Adalimumab for induction of remission in Crohn's disease. Cochrane Database Syst Rev. 2019. https://doi.org/ 10.1002/14651858.CD012878.pub2.

22. Gregor JC, McDonald JW, Klar N, et al. An evaluation of utility measurement in Crohn's disease. Inflamm Bowel Dis. 1997;3:265-76.

23. Williet N, Sandborn WJ, Peyrin-Biroulet L. Patientreported outcomes as primary end points in clinical trials of inflammatory bowel disease. Clin Gastroenterol Hepatol. 2014;12(1246-56):e6.

24. Janssen MF, Pickard AS, Golicki D, et al. Measurement properties of the EQ-5D-5L compared to the EQ-5D-3L across eight patient groups: a multicountry study. Qual Life Res. 2013;22:1717-27.

25. Reilly MC, Zbrozek AS, Dukes EM. The validity and reproducibility of a work productivity and activity impairment instrument. Pharmacoeconomics. 1993;4:353-65. 
26. Reilly MC, Gerlier L, Brabant Y, Brown M. Validity, reliability, and responsiveness of the work productivity and activity impairment questionnaire in Crohn's disease. Clin Ther. 2008;30:393-404.

27. Reilly Associates. Work productivity and activity questionnaire specific health problem V2.0 (WPAISHP). 2010. http://www.reillyassociates.net/WPAI_ SHP.html. Cited 15 June 2020.

28. Khan I, Sarker SJ, Hackshaw A. Smaller sample sizes for phase II trials based on exact tests with actual error rates by trading-off their nominal levels of significance and power. Br J Cancer. 2012;107: 1801-9.

29. Sandborn WJ, Reilly MC, Brown CJ, Brabant Y, Gerlier LC. Minimally important difference for WPAI:CD scores: defining relevant impact on work productivity in active Crohn's disease. Am J Gastroenterol. 2007;102:S472.

30. Byron C, Cornally N, Burton A, Savage E. Challenges of living with and managing inflammatory bowel disease: a meta-synthesis of patients' experiences. J Clin Nurs. 2020;29:305-19.

31. Garcia-Sanjuan S, Lillo-Crespo M, Richart-Martinez M, Sanjuan Quiles A. Understanding life experiences of people affected by Crohn's disease in Spain. A phenomenological approach. Scand J Car Sci. 2018;32:354-62.
32. Loftus EV, Feagan BG, Colombel JF, et al. Effects of adalimumab maintenance therapy on health-related quality of life of patients with Crohn's disease: patient-reported outcomes of the CHARM trial. Am J Gastroenterol. 2008;103:3132-41.

33. Louis E, Lofberg R, Reinisch W, et al. Adalimumab improves patient-reported outcomes and reduces indirect costs in patients with moderate to severe Crohn's disease: results from the CARE trial. J Crohns Colitis. 2013;7:34-43.

34. Colombel JF, Reinisch W, Mantzaris GJ, et al. Randomised clinical trial: deep remission in biologic and immunomodulator naive patients with Crohn's disease-a SONIC post hoc analysis. Aliment Pharmacol Ther. 2015;41:734-46.

35. Vermeire S, Loftus EV Jr, Colombel JF, et al. Longterm efficacy of vedolizumab for Crohn's disease. J Crohns Colitis. 2017;11:412-24.

36. Feagan BG, Sandborn WJ, Wolf DC, et al. Randomised clinical trial: improvement in health outcomes with certolizumab pegol in patients with active Crohn's disease with prior loss of response to infliximab. Aliment Pharmacol Ther. 2011;33: 541-50.

37. Sands BE, Han C, Gasink C, et al. The effects of ustekinumab on health-related quality of life in patients with moderate to severe Crohn's disease. J Crohns Colitis. 2018;12:883-95. 